\title{
Efecto del tiempo de Cocción del Zapallo (Cucurbita maxima) y la adición de Glucosa Oxidasa en el Aumento de Almidón Resistente del Pan de Molde
}

\author{
Bárbara L. Tasiguano1, Camila Villarreal ${ }^{1}$, Marcio Schmiele ${ }^{2}$ y Maria G. Vernaza ${ }^{1 *}$ \\ (1) Ingeniería en Alimentos. Colegio Politécnico, Universidad San Francisco de Quito, Círculo de Cumbayá, P.O. Box \\ 17-12-841, Quito, Ecuador. (e-mail: batotasi@hotmail.com; cami.villarreal05@gmail.com; mgvernaza@usfq.edu.ec) \\ (2) Engenharia de Alimentos. Instituto de Ciência e Tecnologia, Universidade Federal dos Vales do Jequitinhonha e \\ Mucuri, Diamantina, Minas Gerais, Brasil. (e-mail: marcio.sc@ict.ufvjm.edu.br)
}

* Autor a quien debe ser enviada la correspondencia

Recibido Ago. 3, 2018; Aceptado Oct. 10, 2018; Versión final Nov. 23, 2018, Publicado Jun. 2019

\begin{abstract}
Resumen
Se elaboraron panes de molde con sustitución de harina de trigo por harina de zapallo (13\%), siguiendo un diseño central compuesto rotacional $2^{2}$. Las variables independientes fueron glucosa oxidasa $(0-20 \mathrm{ppm})$ y tiempo de cocción del zapallo antes de ser molido para la obtención de harina (0-20 min). Las variables dependientes fueron: volumen específico, color instrumental y contenido de almidón resistente. Se caracterizaron las mezclas de harinas en Mixolab y se realizó una prueba afectiva de aceptación. Los resultados indican que a mayor tiempo de cocción y valores medios de enzima aumenta la estabilidad de la masa. El debilitamiento de las proteínas aumentó con valores medios de enzima y mayor tiempo de cocción, mientras que la retrogradación del almidón disminuyó en las mismas condiciones. El volumen específico y el contenido de almidón resistente aumentaron con mayor tiempo de cocción. No hubo diferencia estadística significativa sensorial entre tratamientos.
\end{abstract}

Palabras clave: zapallo; Cucurbita maxima; glucosa oxidasa; almidón resistente; tiempo de cocción

\section{Effect of Cooking Time of Pumpkin (Cucurbita maxima) and the addition of Glucose Oxidase on the Increase of Resistant Starch in Loaf Bread}

\begin{abstract}
Bread was made with wheat flour substitution for pumpkin flour (13\%), following a central rotational composite design $2^{2}$. The independent variables were glucose oxidase $(0-20 \mathrm{ppm})$ and cooking time of pumpkin before being ground to flour (0-20 $\mathrm{min}$ ). The dependent variables were specific volume, instrumental color and content of resistant starch. The mixtures of flours were characterized in Mixolab and an acceptance test was carried out for sensory evaluation. The results showed that a longer cooking time and average enzyme values increase the stability of the dough. The weakening of the proteins increased with average enzyme values and longer cooking time, while retrogradation of the starch decreased under the same conditions. The specific volume and content of resistant starch increased with longer cooking time. There was no significant statistical difference between treatments in the sensory evaluation analysis.
\end{abstract}

Keywords: pumpkin; Cucurbita maxima; glucose oxidase; resistant starch; cooking time 


\section{INTRODUCCIÓN}

Una dieta saludable debe contener cantidades adecuadas de lípidos, proteínas y carbohidratos denominados macronutrientes, al igual que vitaminas y minerales que se conocen como micronutrientes (Webb, 2007). En la actualidad, el mundo está atravesando por cambios en los hábitos alimenticios donde, una alimentación rica en fibras se considera como la mejor opción. Lastimosamente en el Ecuador, este cambio aún no ha sido aceptado por toda la sociedad, pues en el año 2011 enfermedades como diabetes, hipertensión y cardiovasculares fueron las principales causas de muerte (INEC, 2014). En el Ecuador, 1 persona de cada 1000 tiene un consumo adecuado de fibra (Freire, et al., 2013). Como se puede observar, esta cifra es muy baja y en lugar de tener una alimentación nutritiva, se asocia con un alto consumo de carbohidratos refinados y bajo consumo de frutas y verduras.

El zapallo pertenece a la familia de las Cucurbitáceas, crece en zonas de climas calurosos con veranos largos y necesita de abundante agua (Rey, 2016). Las especies de la familia Cucurbitaceae son de gran importancia económica a nivel mundial, porque están presentes en continentes como América, Europa y Asia. Se encuentran entre las familias de plantas más importantes que proveen al hombre de productos alimenticios con propiedades digestivas y nutritivas que aportan al organismo fibra (Rodríguez-Manrique, Alvis-Bermudez y Cohen-Manrique, 2018). La especie Cucurbita maxima parece tener su origen en América, concretamente en zonas próximas a México, donde se han encontrado rastros con una antigüedad superior a los 10.000 años A.C. En países latinoamericanos se tiene una alta producción, tan solo en el Ecuador la producción de zapallo en el año 2014 fue de 3.290 toneladas (FAO, 2016). Existen alimentos que contribuyen a la ingesta de nutrientes como los productos horneados, especialmente el pan ya que durante el transcurso de los años ha probado ser la base de la alimentación humana, debido a su bajo costo y facilidad de elaboración. Por lo tanto, es elegido continuamente para incrementar su valor nutricional y ser llevado a toda la sociedad (Kundu, et al., 2012).

La porción de almidón que no puede ser digerida por enzimas como amilasa se conoce como almidón resistente. Este tipo de almidón no es digerido en el intestino delgado por lo que ingresan al intestino grueso donde se vuelven sustratos disponibles para la fermentación microbiana. El producto de esta fermentación es la producción de ácidos grasos de cadena corta, los cuales tienen beneficios para la salud humana como: reducción de precursores de cáncer de colon, disminución de la respuesta glicémica e incremento de microrganismos beneficiosos de la flora intestinal. Por otro lado, el almidón resistente también es considerado beneficioso para la salud por sus efectos similares a los que presenta una fibra alimentaria (Birt, 2013). Actualmente existen cinco tipos de almidón resistente. El tipo I proviene de granos y semillas enteros que impiden el ingreso de agua a la matriz del almidón por lo que no se tienen las condiciones adecuadas para que el almidón se hidrolice. A su vez, la pared celular del material crea una barrera que previene a las enzimas hidrolizar el almidón. El pan hecho con granos enteros de trigo y pastas de trigo durum son ejemplos de almidón resistente tipo I (Birt, 2013). El tipo II proviene del almidón de papa no cocinada, bananas verdes o maíz con alta cantidad de amilasa.

Algunos de estos una vez que son cocinados se vuelven digeribles como resultado de la gelatinización del almidón, para contrarrestar este efecto se debe cocinar bajo su temperatura de gelatinización y se mantiene la estructura cristalina del almidón haciendo que permanezca resistente a la hidrólisis enzimática (Birt, 2013). El tipo III se conoce también como almidón retrogradado. Las moléculas de amilosa tienen estructuras lineales que tienden a formar dobles hélices, particularmente a temperaturas de refrigeración $\left(4-5^{\circ} \mathrm{C}\right)$. La doble hélice que se forma no se acopla a sitios de reconocimiento enzimático de la amilasa por lo tanto no puede ser hidrolizado (Birt, 2013). El tipo IV, es el almidón modificado químicamente mediante adición de derivados químicos, que restringen parcialmente la hidrólisis de la molécula de almidón (Birt, 2013) y el tipo V se obtiene cuando el almidón interactúa físicamente con los lípidos, la amilosa y amilopectina formando complejos helicoidales simples con ácidos grasos lineares y alcoholes grasos. Cuando la cadena de almidón lineal está en una estructura helicoidal, se impide el enlazamiento del almidón y la hidrólisis por la amilasa. Además, el complejo de amilosa-lípido también enreda las moléculas de amilopectina, restringiendo el hinchamiento de los gránulos de almidón y la hidrólisis enzimática (Birt, 2013).

El uso de fibras alimenticias en la panificación provoca el debilitamiento de la red de gluten. En este sentido, una de las enzimas que se utiliza en panificación es la glucosa oxidasa, que actúa en presencia de oxígeno, donde se cataliza la oxidación de $\alpha$-D-glucosa a $\alpha$-D-gluconolactona y peróxido de hidrogeno $\left(\mathrm{H}_{2} \mathrm{O}_{2}\right)$. Diferentes investigaciones han determinado que no se conoce completamente el mecanismo por el cual la glucosa oxidasa mejora la calidad del pan; sin embargo, se plantea que el peróxido de hidrogeno $\left(\mathrm{H}_{2} \mathrm{O}_{2}\right)$ producido en la reacción de la enzima puede causar la oxidación de los grupos sulfhidrilo libres de las proteínas del gluten, además participa en la gelación de las pentosas solubles, lo que puede ayudar a modificar las propiedades reológicas en la masa elaborada (Steffolani, 2010). El tratamiento de harina de trigo con glucosa oxidasa puede ayudar en el entrecruzamiento de la fracción de proteínas solubles mediante 
enlaces disulfuro. También se ha demostrado que al añadir esta enzima se produce un cambio en la textura de la masa, lo cual afecta su consistencia y aumenta el volumen del pan. Es importante considerar que el efecto de la enzima puede depender de la dosis y de la calidad de la harina utilizada (Castelli, 2002). Por todo lo establecido anteriormente, el objetivo fue desarrollar un pan de molde elaborado a partir de la sustitución parcial de harina de trigo por harina de zapallo cocinada a diferentes tiempos y con adición de la enzima glucosa oxidasa para evaluar los beneficios que estos pueden otorgar al producto final.

\section{METODOLOGÍA}

Se utilizaron zapallos (Cucurbita maxima) de aproximadamente $10 \mathrm{~kg}$ y en estado de inmadurez. Se utilizó harina de trigo para panificación (Planiplus, Moderna Alimentos con la siguiente caracterización: 11\% proteína, $65.5 \%$ absorción de agua, 5 min de estabilidad, $36 \%$ gluten húmedo, $10 \%$ gluten seco y 280 s Falling Number). La glucosa oxidasa fue proporcionada por Granotec-Ecuador.

\section{Elaboración de la Harina de Zapallo}

Para la elaboración de harina de zapallo se siguió la metodología descrita por Alava (2007). En primer lugar, los frutos se desinfectaron con una solución de hipoclorito de sodio (100 ppm) y se enjuagaron con agua hasta un remanente de cloro de $5 \mathrm{ppm}$. Se separó la pulpa de la cáscara y se rebanó en rodajas de $3.5 \mathrm{~mm}$, las que se cocinaron a $92^{\circ} \mathrm{C}$ (temperatura de ebullición en Cumbayá, Quito - Ecuador) siguiendo los tiempos establecidos en el diseño experimental (Tabla 1). Posteriormente la pulpa cocinada se secó a $80^{\circ} \mathrm{C}$ por $8 \mathrm{~h}$. Finalmente, se trituró el producto seco y se pasó por un tamiz con abertura de $0.420 \mathrm{~mm}$ para la obtención de la harina.

\section{Elaboración de Pan de Molde}

Para la obtención de pan de zapallo se utilizó el método de masa sin tiempo con la siguiente formulación base: $87 \%$ harina de trigo, $13 \%$ harina de zapallo (cocinada a diferentes tiempos), $60 \%$ agua, $2 \%$ sal, $4 \%$ levadura, $5 \%$ grasa, $5 \%$ azúcar, $0.7 \%$ mejorador. En primer lugar, se realizó la mezcla de los ingredientes durante $6 \mathrm{~min}$ hasta el desarrollo óptimo de la red de gluten. Posteriormente se formaron panes de $420 \mathrm{~g}$ y se llevaron a la cámara de fermentación en moldes de aluminio durante $50 \mathrm{~min}\left(40^{\circ} \mathrm{C} / 85 \%\right.$ humedad relativa). Transcurrido este tiempo se hornearon los panes a $170^{\circ} \mathrm{C}$ por $20 \mathrm{~min}$ en horno eléctrico. El pan horneado se almacenó a temperatura ambiente en bolsas de polietileno durante $24 \mathrm{~h}$ para su posterior análisis.

\section{Diseño Experimental}

Para la optimización de la sustitución de la harina de trigo por harina de zapallo en la obtención de pan de molde se utilizó un diseño central compuesto rotacional (DCCR) $2^{2}$ donde los factores de estudio fueron la adición de glucosa oxidasa $\left(X_{1}\right)$ de 0 a 20ppm (Steffolani, 2010) y tiempo de cocción $\left(X_{2}\right)$ de 0 a 20 minutos (Rodríguez-Ambriz, et al.,2007). Los rangos establecidos de cada variable fueron analizados en pruebas preliminares. Se realizaron un total de 12 ensayos, siendo 4 factoriales (combinaciones entre los niveles -1 y +1 ), 4 axiales (una variable en el nivel $\pm \alpha$, otra en 0 ) y cuatro centrales (las dos variables en el nivel 0), con un valor $\pm \alpha$ de 1.4142 para garantizar la rotabilidad del diseño (Myers y Anderson-Cook, 2016).

\section{Métodos Analíticos}

Para analizar el comportamiento de las masas al adicionar la harina de zapallo cocinada a diferentes tiempos y enzima glucosa oxidasa se realizaron análisis de reología mediante sus propiedades termomecánicas utilizando el equipo Mixolab ${ }^{\mathrm{TM}}$ (método AACC 54-60.01) (AACCI, 2010) donde se utilizó el protocolo de Mixolab Estándar (Chopin, Francia). Los parámetros estudiados fueron: absorción de agua $(\mathrm{g} / 100 \mathrm{~g})$, tiempo de desarrollo de la masa ( $\mathrm{min})$, estabilidad $(\mathrm{min}), \mathrm{C} 1(\mathrm{Nm}), \mathrm{C} 2(\mathrm{Nm}), \mathrm{C} 2-\mathrm{C} 1(\mathrm{Nm}), \mathrm{C} 3(\mathrm{Nm}), \mathrm{C} 3-\mathrm{C} 2(\mathrm{Nm}), \mathrm{C} 4$ $(\mathrm{Nm})$, C4-C3 $(\mathrm{Nm})$, C5 $(\mathrm{Nm})$ y C5-C4 $(\mathrm{Nm})$. Los análisis se realizaron por duplicado. Los panes obtenidos fueron caracterizados cuanto a su pH siguiendo el método 02-52.01 de la AACCI (2010), actividad acuosa utilizando un determinador eléctrico Hygrolab C1 (Hygrolab C1, Rotronic, Bassersdorf, Switzerland), humedad por estufa según el método $44-15.02$ (AACCI, 2010) de y volumen específico según el método 55 - 50.01 (AACCl, 2010). Además, se analizó el color instrumental con la ayuda de un colorímetro Chroma Meter CR400 Konica Minolta, usando el espacio rectangular Hunter $L^{*}, C^{*}$, h y se determinó el contenido de almidón resistente según el método $32-40.01$ de la AACCI (2010). Los análisis se realizaron por duplicado.

\section{Optimización y caracterización de los puntos óptimos}

Para la optimización de las formulaciones se utilizó la función de deseabilidad (FD) mediante el software Design Expert 10, donde se asignó importancias y se estableció objetivos, de maximización, minimización y mantención en los rangos estudiados de cada variable de respuesta (Derringer y Suich,1980). En el proceso 
de optimización se analizaron todos los tratamientos y a partir de los valores resultantes de deseabilidad se eligieron los dos mejores tratamientos que presentaron los mayores valores de deseabilidad. A partir de estos resultados se realizó la evaluación sensorial. Las formulaciones optimizadas fueron caracterizadas según su composición proximal: cenizas (Calcinación en mufla, método gravimétrico, AOAC 923.03), proteína (Método de Kjehdahl AOAC 991.20), grasa (Soxhlet. Método AOAC 920.39), humedad (estufa según el método AACCI $44-15.02)$ y carbohidratos totales (Diferencia).

\section{Evaluación Sensorial}

Para el análisis sensorial se utilizó una prueba afectiva de aceptación con la aplicación de una escala hedónica de 9 puntos siendo 1 = "me disgusta muchísimo" y 9 = "me gusta muchísimo". Los atributos analizados fueron color, aroma, sabor, textura y apariencia global. La evaluación fue realizada a 72 jueces no entrenados entre 18 y 55 años.

\section{Análisis del alveolado de los tratamientos óptimos}

Para analizar el alveolado de la miga se escanearon 4 rodajas de diferentes panes en un escáner Epson L355, con una resolución de 600 dpi. Las imágenes fueron procesadas a través del programa ImageJ versión 143 analizando la imagen de la miga, según la metodología de Correa (2012). Las características analizadas de la miga fueron: área total de la rodaja $(\mathrm{cm} 2)$, perímetro $(\mathrm{cm})$, circularidad, número de alveolos por $\mathrm{cm}^{2}$, tamaño medio de los alvéolos ( $\mathrm{mm} 2$ ) y área media de los alveolos (\%).

\section{Análisis estadístico}

Los resultados obtenidos en el CCDR se evaluaron mediante la Metodología de Superficie de Respuesta. Los coeficientes de regresión utilizados en los modelos matemáticos fueron validados por el análisis de varianza (ANOVA) $(p<0.10)$ y con un $R^{2}$ mínimo de 0.70 . Se utilizaron modelos matemáticos con valores codificados siendo $X_{1}$ y $X_{2}$ la adición de la enzima y el tiempo de cocción, respectivamente. Se utilizó el software Statistica. Los resultados de la evaluación sensorial se analizaron en el software Minitab versión 16 en base a un Diseño Latino Cuadrado (DLC) en el que se bloqueó el orden de presentación de los tratamientos y el efecto de los panelistas. El nivel de significancia para los análisis se estableció en un valor de 0.05 . Finalmente, los resultados fisicoquímicos de los tratamientos optimizados fueron estudiados por un análisis de varianza simple (ANOVA) $(\mathrm{p}<0.05)$.

\section{RESULTADOS Y DISCUSION}

Con los resultados obtenidos para la Estabilidad, Debilitamiento de las proteínas (C2), Estabilidad del gel caliente formado (C4), Tendencia a la retrogradación del almidón (C5-C4), Luminosidad ( $\left.L^{*}\right)$, Volumen específico y Contenido de almidón resistente fue posible obtener superficies de respuesta $(p<0.10$ y R2>0.70).

\section{Estabilidad}

De acuerdo con el modelo matemático obtenido para la respuesta Estabilidad (Tabla 4), la glucosa oxidasa presenta una función cuadrática negativa por lo cual se observa la tendencia a una curvatura en esta variable; es decir, que a medida que se aumenta la cantidad de la enzima, la estabilidad crecerá y luego volverá a disminuir (Figura 1a). Por otro lado, en este modelo el tiempo de cocción afecta en mayor proporción a la variable estabilidad. La interacción entre ambas variables independientes tiende a aumentar el valor de la estabilidad, esto significa que al aumentar el tiempo de cocción se contrarresta el efecto negativo que puede otorgar la glucosa oxidasa en la estabilidad. Cuando la enzima GO se encuentra en presencia del oxígeno se produce una reacción de oxidación, donde se forma peróxido de hidrógeno $\left(\mathrm{H}_{2} \mathrm{O}_{2}\right)$, el cual oxida indirectamente los grupos sulfhidrilo del gluten, ocasionando que se transformen en enlaces disulfuro, lo cual fortalece la red de gluten (Steffolani, 2010). Sin embargo, al colocar una mayor cantidad de GO la estabilidad disminuye considerablemente, debido a que la enzima funciona de mejor manera en masas que contengan harinas con bajo nivel proteico o débiles (Bock, 2015), en este estudio se utilizó harina de trigo fuerte, por lo que no se pudo apreciar el efecto deseado. En cuanto al tiempo de cocción, mientras éste sea mayor, la cantidad de almidón gelatinizado es mayor en la masa, aumentando de esta forma la viscosidad del fluido viscoelástico, (Onyango,2016), mejorando así la estabilidad al amasado.

\section{C2 (Debilitamiento de las Proteínas)}

El modelo matemático obtenido para C2 fue lineal (Tabla 4 y Figura 1b). El tiempo de cocción del zapallo influye en el incremento de valor de C2 debido a que el almidón presente se gelatiniza, obteniendo una harina de zapallo pre gelatinizada. Al sustituir parcialmente la harina de trigo con esta harina se incrementa la cantidad de almidón gelatinizado (Onyango, 2016) que hace que se absorba una mayor cantidad de agua en el proceso de cocción (horneado); el almidón toma el agua del gluten lo que producirá una red menos 
hidratada (Wang, 2003). Se desea que el valor de C2 sea lo más cercano a 0.5 (Nm) para lograr una masa con tenacidad adecuada y un pan con mayor volumen (Pineda, 2013). En este estudio los valores máximos de C2 se encuentran en los puntos centrales (Figura 1b).
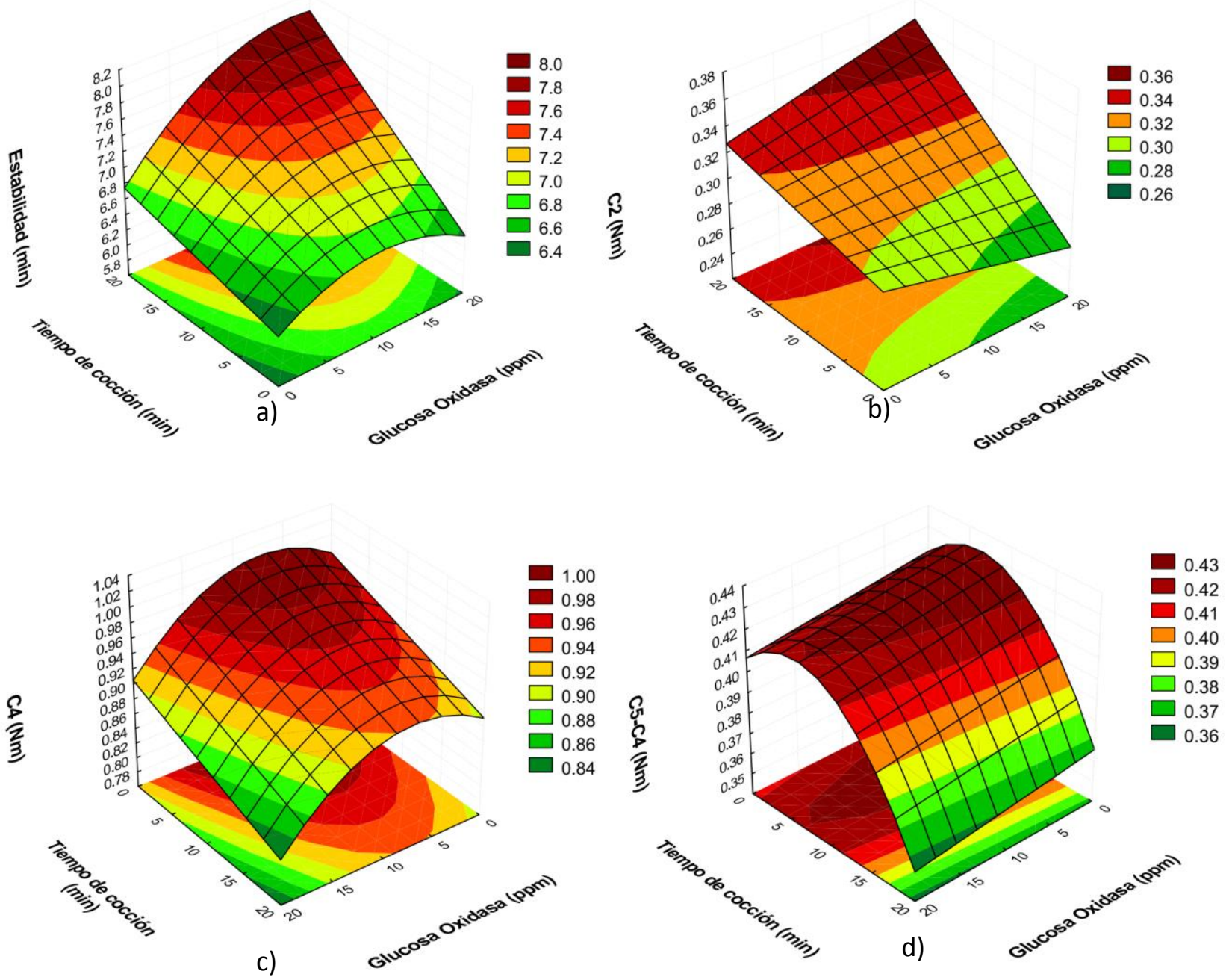

Fig. 1: Superficies de respuesta para: a) Estabilidad (min), b) C2(Nm), c) C4(Nm), d) C5-C4 (Nm)

\section{C4 (Estabilidad del gel caliente formado)}

En el modelo matemático del parámetro C4 (Tabla 4), se observa que la glucosa oxidasa determina la curvatura de la parábola. Como se puede observar en la Figura 1c, el parámetro C4 llega a su punto más elevado al momento en que la glucosa oxidasa se encuentra entre los valores medios; una vez que pasa de estos valores, el parámetro C4 decae dando un efecto negativo a la estabilidad del gel. Los pentosanos presentes en la harina de trigo tienen la habilidad de gelificar en presencia de radicales libres, por lo que la $\mathrm{GO}$ al momento de ser catalizada produce $\mathrm{H}_{2} \mathrm{O}_{2}$ que facilita la producción de estos radicales y por ende una mayor estabilidad del gel formado (Vemulapalli, Miller y Hoseney, 1998). Adicionalmente, se puede mencionar que en este caso la variable de tiempo de cocción afecta de manera mínima a la variable dependiente y a medida que éste disminuye favorece al incremento de C4.

\section{C5-C4 (Retrogradación del Almidón)}

El modelo matemático obtenido para C5-C4 fue cuadrático (Tabla 4), el cual se vio únicamente afectado por el tiempo de cocción. La retrogradación del almidón consiste en la reasociación de las cadenas de amilosa y amilopectina, eliminando agua, disminuyendo la vida útil del pan y aumentando su dureza, por lo que se desea valores bajos de (C5-C4). A medida que el tiempo de cocción aumenta se forma una mayor cantidad de almidón resistente tipo III, el cual ayuda a retener agua, disminuyendo la retrogradación del almidón (Loor, 2008). Una alta retrogradación del almidón tiene un efecto negativo en la calidad de ciertos productos alimenticios, en el pan ocurre un fenómeno conocido como envejecimiento el cual produce dureza y menor tiempo de vida útil, lo cual repercute en la aceptación del consumidor y en la industria de panificación (Fu et al., 2015). Los valores mínimos de C5-C4 corresponden a los puntos extremos en la Figura 1d. 
Tabla 1: Resultados del Análisis de Mixolab de las mezclas de harinas

\begin{tabular}{|c|c|c|c|c|c|c|c|}
\hline & \multicolumn{2}{|c|}{ Variables independientes } & \multicolumn{5}{|c|}{ Variables de respuesta } \\
\hline Trat & $\begin{array}{c}\text { Glucosa Oxidasa } \\
\text { (ppm) } X_{1}\end{array}$ & $\begin{array}{c}\text { Tiempo de } \\
\text { cocción (min) } X_{2}\end{array}$ & $\begin{array}{l}\text { Abs. Agua } \\
\text { (g/100 g) }\end{array}$ & $\begin{array}{c}T \text { desarrollo } \\
\text { de masa (min) }\end{array}$ & $\begin{array}{l}\text { Estabilidad } \\
\quad \text { (min) }\end{array}$ & $\mathrm{C1}(\mathrm{Nm})$ & $\mathrm{C2}(\mathrm{Nm})$ \\
\hline T1 & 3 & 3 & 75.00 & $5.12 \pm 0.07$ & $6.55 \pm 0.00$ & $1.10 \pm 0.00$ & $0.31 \pm 0.00$ \\
\hline T2 & 17 & 3 & 75.00 & $5.01 \pm 0.23$ & $6.75 \pm 0.25$ & $1.07 \pm 0.03$ & $0.30 \pm 0.01$ \\
\hline T3 & 3 & 17 & 76.10 & $4.83 \pm 0.18$ & $6.95 \pm 0.31$ & $1.13 \pm 0.01$ & $0.33 \pm 0.00$ \\
\hline T4 & 17 & 17 & 74.80 & $5.30 \pm 0.42$ & $7.73 \pm 0.04$ & $1.14 \pm 0.01$ & $0.35 \pm 0.01$ \\
\hline T5 & 0 & 10 & 72.30 & $5.00 \pm 0.07$ & $7.20 \pm 0.31$ & $1.10 \pm 0.02$ & $0.31 \pm 0.00$ \\
\hline T6 & 20 & 10 & 72.20 & $5.04 \pm 0.09$ & $6.51 \pm 0.27$ & $1.11 \pm 0.01$ & $0.29 \pm 0.00$ \\
\hline T7 & 10 & 0 & 68.00 & $5.20 \pm 0.11$ & $6.65 \pm 0.14$ & $1.09 \pm 0.02$ & $0.27 \pm 0.01$ \\
\hline T8 & 10 & 20 & 73.00 & $5.48 \pm 0.35$ & $7.84 \pm 0.16$ & $1.11 \pm 0.01$ & $0.34 \pm 0.00$ \\
\hline T9 & 10 & 10 & 73.50 & $4.96 \pm 0.13$ & $6.23 \pm 0.04$ & $1.10 \pm 0.01$ & $0.28 \pm 0.00$ \\
\hline T10 & 10 & 10 & 72.30 & $5.19 \pm 0.20$ & $7.31 \pm 0.27$ & $1.09 \pm 0.01$ & $0.31 \pm 0.00$ \\
\hline T11 & 10 & 10 & 71.70 & $4.90 \pm 0.11$ & $7.19 \pm 0.02$ & $1.13 \pm 0.02$ & $0.32 \pm 0.01$ \\
\hline T12 & 10 & 10 & 70.90 & $5.32 \pm 0.07$ & $7.15 \pm 0.25$ & $1.11 \pm 0.00$ & $0.31 \pm 0.01$ \\
\hline & \multicolumn{7}{|c|}{ Variables de Respuesta } \\
\hline Trat & $\begin{array}{c}\text { C1-C2 } \\
(\mathrm{Nm})\end{array}$ & C3 (Nm) & $\begin{array}{l}\text { C3-C2 } \\
(\mathrm{Nm})\end{array}$ & $\mathrm{C4}(\mathrm{Nm})$ & $\begin{array}{l}\text { C3-C4 } \\
(\mathrm{Nm})\end{array}$ & C5 $(\mathrm{Nm})$ & $\begin{array}{l}\text { C5-C4 } \\
(\mathrm{Nm})\end{array}$ \\
\hline $\mathrm{T} 1$ & $0.79 \pm 0.00$ & $1.25 \pm 0.00$ & $0.94 \pm 0.01$ & $0.95 \pm 0.03$ & $0.30 \pm 0.03$ & $1.37 \pm 0.04$ & $0.43 \pm 0.00$ \\
\hline T2 & $0.78 \pm 0.01$ & $1.23 \pm 0.00$ & $0.93 \pm 0.01$ & $0.92 \pm 0.01$ & $0.31 \pm 0.01$ & $1.35 \pm 0.03$ & $0.44 \pm 0.02$ \\
\hline T3 & $0.80 \pm 0.01$ & $1.17 \pm 0.01$ & $0.84 \pm 0.00$ & $0.83 \pm 0.01$ & $0.34 \pm 0.00$ & $1.21 \pm 0.01$ & $0.38 \pm 0.00$ \\
\hline T4 & $0.79 \pm 0.00$ & $1.22 \pm 0.01$ & $0.87 \pm 0.00$ & $0.90 \pm 0.02$ & $0.32 \pm 0.01$ & $1.29 \pm 0.00$ & $0.39 \pm 0.02$ \\
\hline T5 & $0.79 \pm 0.01$ & $1.24 \pm 0.01$ & $0.93 \pm 0.01$ & $0.94 \pm 0.01$ & $0.29 \pm 0.02$ & $1.37 \pm 0.01$ & $0.43 \pm 0.02$ \\
\hline T6 & $0.81 \pm 0.01$ & $1.21 \pm 0.00$ & $0.91 \pm 0.00$ & $0.89 \pm 0.01$ & $0.31 \pm 0.01$ & $1.30 \pm 0.01$ & $0.41 \pm 0.01$ \\
\hline $\mathrm{T7}$ & $0.81 \pm 0.02$ & $1.30 \pm 0.02$ & $1.03 \pm 0.01$ & $1.01 \pm 0.01$ & $0.29 \pm 0.01$ & $1.41 \pm 0.01$ & $0.40 \pm 0.01$ \\
\hline T8 & $0.77 \pm 0.01$ & $1.29 \pm 0.01$ & $0.95 \pm 0.01$ & $0.97 \pm 0.01$ & $0.32 \pm 0.02$ & $1.34 \pm 0.01$ & $0.38 \pm 0.00$ \\
\hline T9 & $0.82 \pm 0.01$ & $1.19 \pm 0.00$ & $0.90 \pm 0.00$ & $0.86 \pm 0.01$ & $0.33 \pm 0.01$ & $1.27 \pm 0.00$ & $0.41 \pm 0.00$ \\
\hline T10 & $0.78 \pm 0.01$ & $1.23 \pm 0.00$ & $0.92 \pm 0.00$ & $0.93 \pm 0.01$ & $-0.30 \pm .01$ & $1.36 \pm 0.04$ & $0.42 \pm 0.03$ \\
\hline T11 & $0.82 \pm 0.01$ & $1.27 \pm 0.02$ & $0.95 \pm 0.01$ & $0.99 \pm 0.02$ & $0.28 \pm 0.00$ & $1.41 \pm 0.01$ & $0.42 \pm 0.01$ \\
\hline T12 & $0.80 \pm 0.00$ & $1.27 \pm 0.00$ & $0.96 \pm 0.00$ & $0.98 \pm 0.00$ & $0.29 \pm 0.00$ & $1.40 \pm 0.01$ & $0.42 \pm 0.00$ \\
\hline
\end{tabular}

\section{Volumen Específico}

El modelo matemático encontrado para el volumen específico (Tabla 4), muestra que el tiempo de cocción es la variable que tiene un mayor efecto sobre el volumen específico. Como se muestra en la Figura 2a los extremos del paraboloide tuvieron un mayor volumen específico, es decir, al mayor tiempo de cocción y al menor. Por otro lado, se vio un efecto negativo de la enzima, a mayor cantidad de glucosa oxidasa hubo un ligero decremento de volumen específico, debido a que el efecto de la enzima depende de la cantidad utilizada y el tipo de harina, al adicionarla a harinas de buena calidad se produce una masa tenaz y poco extensible, lo que resulta en un pan con bajo volumen (Steffolani, 2010).

Se puede evidenciar el efecto del tiempo de cocción del zapallo porque a medida que transcurre la cocción, el almidón se gelatiniza, lo que aumenta la absorción de agua y es más fácil que sea degradado por enzimas, por lo cual las levaduras tienen más sustratos para la producción de $\mathrm{CO}_{2}$, por lo que se incrementa el volumen del pan en el proceso de fermentación (Rosell, 2011). Se desea maximizar el volumen específico debido a las expectativas del consumidor en la calidad del pan, por lo que se debe tomar en cuenta los valores adecuados tanto de glucosa oxidasa como del tiempo de cocción ya que su interacción produce el efecto esperado en el volumen especifico del producto. 

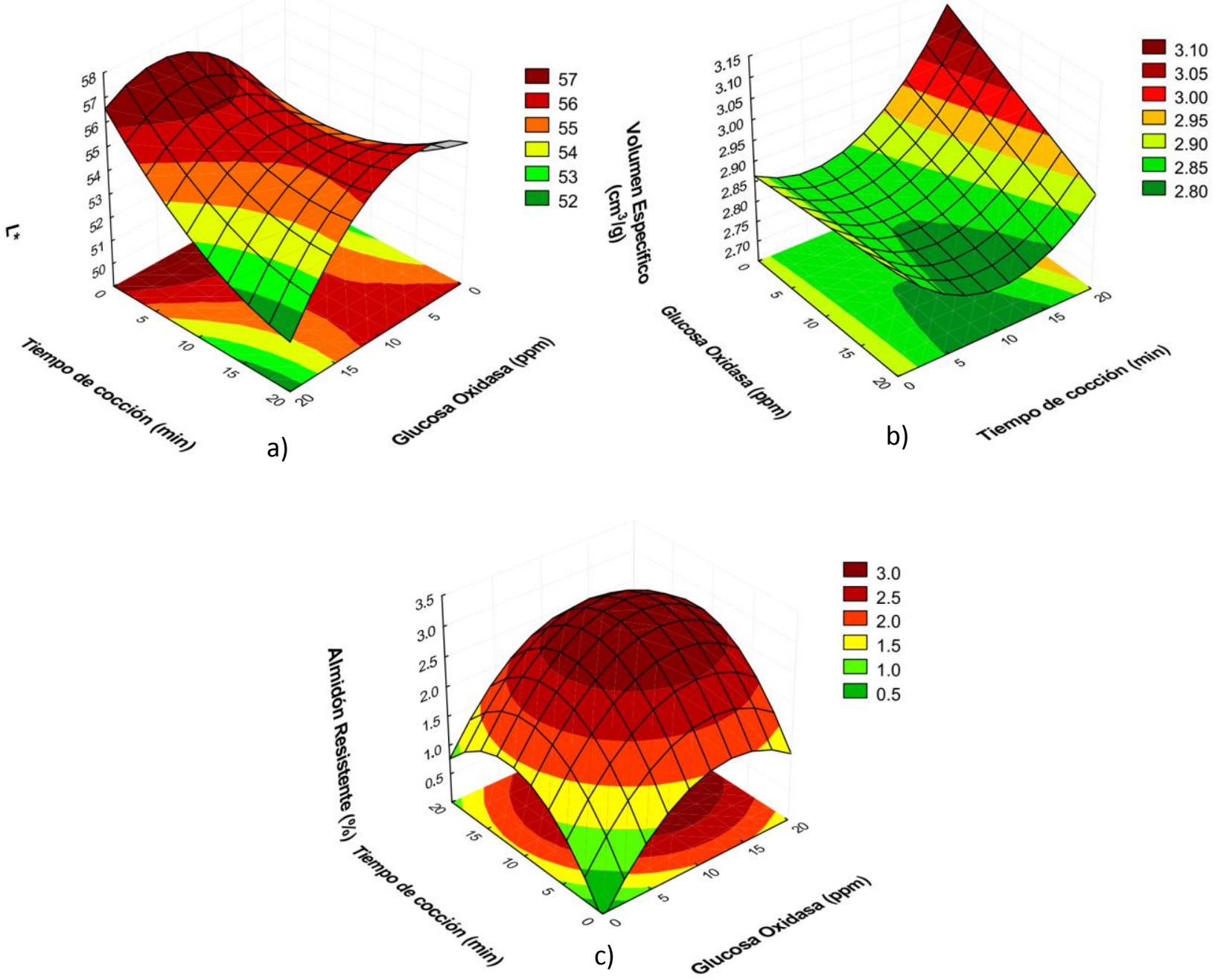

Fig. 2: Superficies de respuesta para: a) $L^{*}$, b) Volumen Específico (cm3/g) y c) Almidón Resistente (\%)

Tabla 2: Resultados de la caracterización físico-química de los panes.

\begin{tabular}{|c|c|c|c|c|c|c|c|}
\hline \multirow{2}{*}{ Tratamientos } & \multirow{2}{*}{$A R(\%)$} & \multirow{2}{*}{$V E\left[\mathrm{~cm}^{3} / \mathrm{g}\right]$} & \multirow{2}{*}{$A w$} & \multirow{2}{*}{$p H$} & \multicolumn{2}{|c|}{$p H$ masas } & \multirow{2}{*}{ Humedad (\%) } \\
\hline & & & & & A.F & $D . F$ & \\
\hline $\mathrm{T} 1$ & $1.09 \pm 0.07$ & $2.86 \pm 0.09$ & $0.93 \pm 0.00$ & $5.36 \pm 0.02$ & $5.51 \pm 0.01$ & $5.37 \pm 0.02$ & $40.28 \pm 0.01$ \\
\hline T2 & $2.87 \pm 0.02$ & $2.79 \pm 0.10$ & $0.94 \pm 0.00$ & $5.43 \pm 0.03$ & $5.55 \pm 0.01$ & $5.38 \pm 0.02$ & $41.59 \pm 0.01$ \\
\hline T3 & $1.19 \pm 0.17$ & $3.05 \pm 0.03$ & $0.94 \pm 0.01$ & $5.42 \pm 0.01$ & $5.63 \pm 0.06$ & $5.30 \pm 0.01$ & $38.63 \pm 0.01$ \\
\hline T4 & $2.88 \pm 0.40$ & $2.93 \pm 0.15$ & $0.94 \pm 0.00$ & $5.52 \pm 0.01$ & $5.52 \pm 0.00$ & $5.23 \pm 0.05$ & $41.13 \pm 0.01$ \\
\hline T5 & $2.01 \pm 0.44$ & $2.81 \pm 0.05$ & $0.93 \pm 0.00$ & $5.49 \pm 0.01$ & $5.64 \pm 0.03$ & $5.36 \pm 0.01$ & $40.48 \pm 0.00$ \\
\hline T6 & $1.93 \pm 0.02$ & $2.81 \pm 0.02$ & $0.93 \pm 0.00$ & $5.46 \pm 0.04$ & $5.63 \pm 0.04$ & $5.36 \pm 0.01$ & $39.70 \pm 0.01$ \\
\hline T7 & $1.03 \pm 0.10$ & $2.90 \pm 0.11$ & $0.93 \pm 0.00$ & $5.47 \pm 0.02$ & $5.58 \pm 0.09$ & $5.32 \pm 0.03$ & $40.42 \pm 0.00$ \\
\hline T8 & $2.70 \pm 0.16$ & $2.95 \pm 0.02$ & $0.93 \pm 0.00$ & $5.51 \pm 0.02$ & $5.61 \pm 0.02$ & $5.18 \pm 0.03$ & $39.36 \pm 0.00$ \\
\hline T9 & $2.77 \pm 0.21$ & $2.81 \pm 0.02$ & $0.94 \pm 0.00$ & $5.52 \pm 0.03$ & $5.66 \pm 0.01$ & $5.48 \pm 0.01$ & $41.07 \pm 0.01$ \\
\hline T10 & $3.06 \pm 0.16$ & $2.81 \pm 0.11$ & $0.93 \pm 0.00$ & $5.47 \pm 0.03$ & $5.59 \pm 0.03$ & $5.42 \pm 0.02$ & $40.35 \pm 0.00$ \\
\hline T11 & $2.87 \pm 0.03$ & $3.09 \pm 0.04$ & $0.94 \pm 0.00$ & $5.47 \pm 0.02$ & $5.55 \pm 0.03$ & $5.18 \pm 0.02$ & $38.94 \pm 0.00$ \\
\hline T12 & $2.78 \pm 0.24$ & $2.76 \pm 0.16$ & $0.94 \pm 0.00$ & $5.51 \pm 0.01$ & $5.51 \pm 0.03$ & $5.23 \pm 0.02$ & $38.94 \pm 0.00$ \\
\hline
\end{tabular}




\section{Almidón Resistente}

El modelo matemático encontrado para el almidón resistente muestra una tendencia cuadrática (Tabla 4). En la Figura $2 b$ se puede observar la superficie de respuesta obtenida y el efecto positivo tanto de la glucosa oxidasa como del tiempo de cocción. Los valores medios presentan el mayor contenido de almidón resistente y los extremos el menor. El almidón resistente tipo III se forma a partir del proceso de retrogradación del almidón, donde se lo expone a bajas temperaturas después de la etapa de gelatinización, lo cual genera que los gránulos de almidón se hidraten hasta perder su estructura por completo, posteriormente estas estructuras se vuelven a asociar (cadenas de amilosa y amilopectina), obteniendo almidón resistente Tipo III (Loor, 2008). Además de los cuatro tipos de almidón resistente ya reportados, recientemente se introdujo un nuevo tipo, almidón resistente tipo $\mathrm{V}$, el cual puede aparecer por la formación del complejo entre amilosa y lípidos que es resistente a la digestión enzimática (Hasjim, Ai y Jane, 2013). En la formulación del pan de este estudio se utiliza $5 \%$ de grasa el cual puede formar este tipo de almidón resistente, ya que el complejo amilosa-lípido se encuentra comúnmente en gránulos de almidón natural y almidón procesado, como es el caso del pan.

El efecto de la glucosa oxidasa se debe al $\mathrm{H}_{2} \mathrm{O}_{2}$, que permite que el gránulo de almidón este mejor hidratado, lo cual genera una gelatinización adecuada y por ende una mejor retrogradación, lo cual resulta en una mayor cantidad de almidón resistente formado (Bock, 2015). En la Figura $2 b$ se observa que los valores más altos de almidón resistente corresponden a los puntos centrales.

Tabla 3: Resultados del análisis de color instrumental

\begin{tabular}{|c|c|c|c|c|c|}
\hline Tratamientos & $L^{*}$ & $a^{*}$ & $b^{*}$ & Hue & Croma \\
\hline T1 & $52.63 \pm 0.86$ & $6.96 \pm 0.17$ & $23.62 \pm 0.38$ & $87.62 \pm 0.04$ & $24.62 \pm 0.37$ \\
\hline T2 & $51.78 \pm 1.36$ & $6.97 \pm 0.14$ & $23.53 \pm 2.04$ & $87.60 \pm 0.18$ & $24.54 \pm 1.98$ \\
\hline T3 & $55.05 \pm 1.39$ & $4.71 \pm 0.31$ & $27.16 \pm 0.65$ & $87.94 \pm 0.05$ & $27.57 \pm 0.63$ \\
\hline T4 & $57.96 \pm 0.88$ & $4.52 \pm 0.14$ & $26.93 \pm 0.79$ & $87.92 \pm 0.06$ & $27.31 \pm 0.78$ \\
\hline T5 & $55.47 \pm 0.68$ & $6.68 \pm 0.17$ & $25.55 \pm 0.36$ & $87.80 \pm 0.03$ & $26.41 \pm 0.37$ \\
\hline T6 & $55.12 \pm 0.99$ & $6.71 \pm 0.16$ & $25.50 \pm 0.61$ & $87.80 \pm 0.05$ & $26.37 \pm 0.61$ \\
\hline T7 & $53.42 \pm 0.92$ & $7.59 \pm 0.20$ & $24.70 \pm 0.79$ & $87.72 \pm 0.08$ & $25.84 \pm 0.81$ \\
\hline T8 & $59.07 \pm 0.98$ & $4.68 \pm 0.14$ & $31.85 \pm 8.75$ & $88.17 \pm 0.29$ & $32.21 \pm 8.70$ \\
\hline T9 & $57.30 \pm 0.84$ & $5.62 \pm 0.17$ & $26.20 \pm 0.44$ & $87.86 \pm 0.04$ & $26.79 \pm 0.45$ \\
\hline T10 & $55.77 \pm 0.86$ & $6.18 \pm 0.21$ & $25.34 \pm 0.76$ & $87.78 \pm 0.07$ & $26.08 \pm 0.78$ \\
\hline T11 & $55.00 \pm 0.78$ & $6.13 \pm 0.16$ & $24.90 \pm 0.50$ & $87.74 \pm 0.05$ & $25.64 \pm 0.51$ \\
\hline T12 & $55.59 \pm 0.83$ & $6.22 \pm 0.21$ & $24.73 \pm 0.36$ & $87.73 \pm 0.03$ & $25.50 \pm 0.37$ \\
\hline
\end{tabular}

\section{Luminosidad $\left(L^{*}\right)$}

El modelo matemático que se obtuvo para el color instrumental (luminosidad) presenta una tendencia cuadrática. En la Figura 2c se puede observar la superficie de respuesta obtenida. A medida que el tiempo de cocción del zapallo aumenta, el valor de $L^{*}$ también lo hace. El zapallo tiene un alto contenido de carotenoides ( $\beta$-caroteno, $\alpha$-caroteno y luteína), que son pigmentos que se mantienen estables a temperatura ambiente; sin embargo, al someterlos a un proceso de calentamiento se vuelven lábiles y se degradan más rápido (Meléndez, Vicario, y Heredia, 2004). Por lo tanto, el valor de L* es mayor, porque al someter al zapallo a un proceso para obtener harina, gran parte de los carotenoides presentes se degradan, por lo que el pan pierde el color característico. De igual forma si el tiempo de cocción disminuye el valor de $L^{*}$ es menor. El efecto de la glucosa oxidasa es también negativo. La degradación de los pigmentos ocurre por la reacción de oxidación de los carotenoides, donde se forman apocarotenoides (compuestos de bajo peso molecular), los cuales pierden su color y propiedades funcionales para la salud (Meléndez, Vicario, y Heredia, 2004). De igual forma si la cantidad de enzima glucosa oxidasa disminuye, el valor de $L^{*}$ es menor.

Humedad, Actividad de agua (Aw) y Ph

Las variables estudiadas no tuvieron efecto significativo en las respuestas de humedad, actividad de agua y $\mathrm{pH}$. Según la norma INEN 2945 que trata sobre los requisitos del pan, la humedad debe estar en un rango de $20 \%$ a $40 \%$, siendo que todos los tratamientos de este estudio están de acuerdo a la norma mencionada. Este parámetro es importante en la conservación de productos de panificación porque influyen en el crecimiento microbiano y velocidad de deterioro, al igual que la Aw. El pH del pan, de la masa antes y después de fermentar se mantuvo en un mismo rango. El pH óptimo de fermentación debe estar entre 5.2 y 5.5 ya que 
si los valores están por fuera de este rango puede darle paso a un aumento excesivo de fuerza en la masa (exceso) o se puede dar paso a que actúen otro tipo de microorganismos que podrían contaminar la masa (Flecha, 2015). Por otro lado, el pH es indispensable para la mayoría de reacciones durante el proceso de panificación y también para el color (Reacción de Maillard).

\section{Función de deseabilidad}

Los parámetros utilizados para obtener la función de deseabilidad se muestran en la Tabla 5. A cada variable se le asignó un objetivo e importancia de acuerdo con lo esperado para la optimización de los tratamientos finales. Las importancias fueron asignadas entre 1-5 siendo 1 menos importante y 5 más importante. Para la primera formulación se maximizaron las siguientes variables: estabilidad, C2, C4, volumen específico y almidón resistente, se minimizó la variable C5-C4 y se mantuvieron en rango la glucosa oxidasa, tiempo de cocción y $L^{*}$. Para la segunda formulación se maximizaron solamente las variables de volumen específico y almidón resistente, las otras variables se mantuvieron en rango. Como resultado los tratamientos óptimos fueron: 916 (8.9 ppm de glucosa oxidasa y 19.2 min de cocción) y 212 (11.4 ppm de glucosa oxidasa y 20 min de cocción). La deseabilidad para 212 y 916 fue de 0.82 y 0.71 respectivamente.

Tabla 4: Modelos matemáticos con variables codificadas obtenidos con el DCCR ( $\mathrm{x}_{1}$ : Glucosa Oxidasa en ppm y x Tiempos de cocción en min)

\begin{tabular}{|c|c|c|}
\hline Variable dependiente & Modelo matemático & $\mathrm{R}^{2} \mathrm{Ajus}$. \\
\hline Estabilidad & $=7.21+0.24 x_{1}-0.19 x_{1}{ }^{2}+0.38 x_{2}+0.15 x_{1} x_{2}$ & 0.98 \\
\hline C2 & $=0.31+0.02 x_{2}+0.01 x_{1} x_{2}$ & 0.89 \\
\hline C4 & $=0.96-0.03 x_{1}-0.03 x_{1}{ }^{2}-0.03 x_{2}$ & 0.70 \\
\hline C5 - C4 & $=0.42-0.01 x_{1}-0.02 x_{2}-0.02 x_{2}{ }^{2}$ & 0.78 \\
\hline Volumen Específico & $=2.80-0.03 x_{1}+0.04 x_{2}+0.07 x_{2}{ }^{2}-0.03 x_{1} x_{2}$ & 0.71 \\
\hline Almidón Resistente & $=2.96+0.45 x_{1}-0.48 x_{1}{ }^{2}+0.31 x_{2}-0.53 x_{2}{ }^{2}$ & 0.77 \\
\hline$L^{*}$ & $=55.30-1.22 x_{1}{ }^{2}-0.53 x_{2}+0.49 x_{2}{ }^{2}-0.99 x_{1} x_{2}$ & \\
\hline
\end{tabular}

\section{Evaluación Sensorial}

Los resultados mostraron que no existió diferencia significativa en ninguno de los atributos medidos tanto para la muestra 916 como para la muestra 212 (Figura 3). En cuanto al color y aroma ambas muestras obtuvieron una calificación promedio ubicada entre los valores 6 y 7 de la escala lo que demuestra que a los jueces les gusto ligeramente este atributo. Ya para los resultados de sabor, textura y aceptación global ambas muestras obtuvieron una calificación promedio ubicada entre los valores 7 y 8 de la escala lo que demuestra que a los jueces les gustó este atributo. En muchos de los comentarios los jueces establecieron que no se percibía una diferencia entre las muestras, esto es una afirmación adecuada pues el tiempo de cocción fue le mismo para ambas y la diferencia recaía en la cantidad de la enzima glucosa oxidasa. Con los resultados obtenidos se pudo mostrar que las cantidades no otorgaron diferencias entre las muestras.

Tabla 5: Objetivos y valores utilizados en la función de deseabilidad para la obtención de los tratamientos optimizados

\begin{tabular}{|l|c|c|c|c|c|c|}
\hline \multicolumn{4}{|c|}{ Optimización de la muestra 212 } & \multicolumn{2}{l|}{ Optimización de la muestra 916 } \\
\hline Parámetro & Objetivo & Importancia & Solución & Objetivo & Importancia & Solución \\
\hline Glucosa Oxidasa $\left(\mathrm{X}_{1}\right)$ & En rango & 3 & 0.20 & En rango & 3 & -0.16 \\
\hline Tiempo de cocción $\left(\mathrm{X}_{2}\right)$ & En rango & 3 & 1.41 & En rango & 3 & 1.30 \\
\hline Estabilidad & Maximizar & 5 & 7.84 & En rango & 5 & 7.64 \\
\hline C2 & Maximizar & 5 & 0.35 & En rango & 5 & 0.34 \\
\hline C4 & Maximizar & 4 & 0.94 & En rango & 4 & 0.94 \\
\hline C5-C4 & Minimizar & 5 & 0.36 & En rango & 5 & 0.37 \\
\hline Volumen Especifico & Maximizar & 5 & 2.97 & Maximizar & 5 & 2.98 \\
\hline Almidón Resistente & Maximizar & 5 & 2.42 & Maximizar & 5 & 2.40 \\
\hline L* & En rango & 3 & 54.82 & En rango & 3 & 54.81 \\
\hline Deseabilidad & \multicolumn{2}{|l|}{0.82} & & 0.71 \\
\hline
\end{tabular}




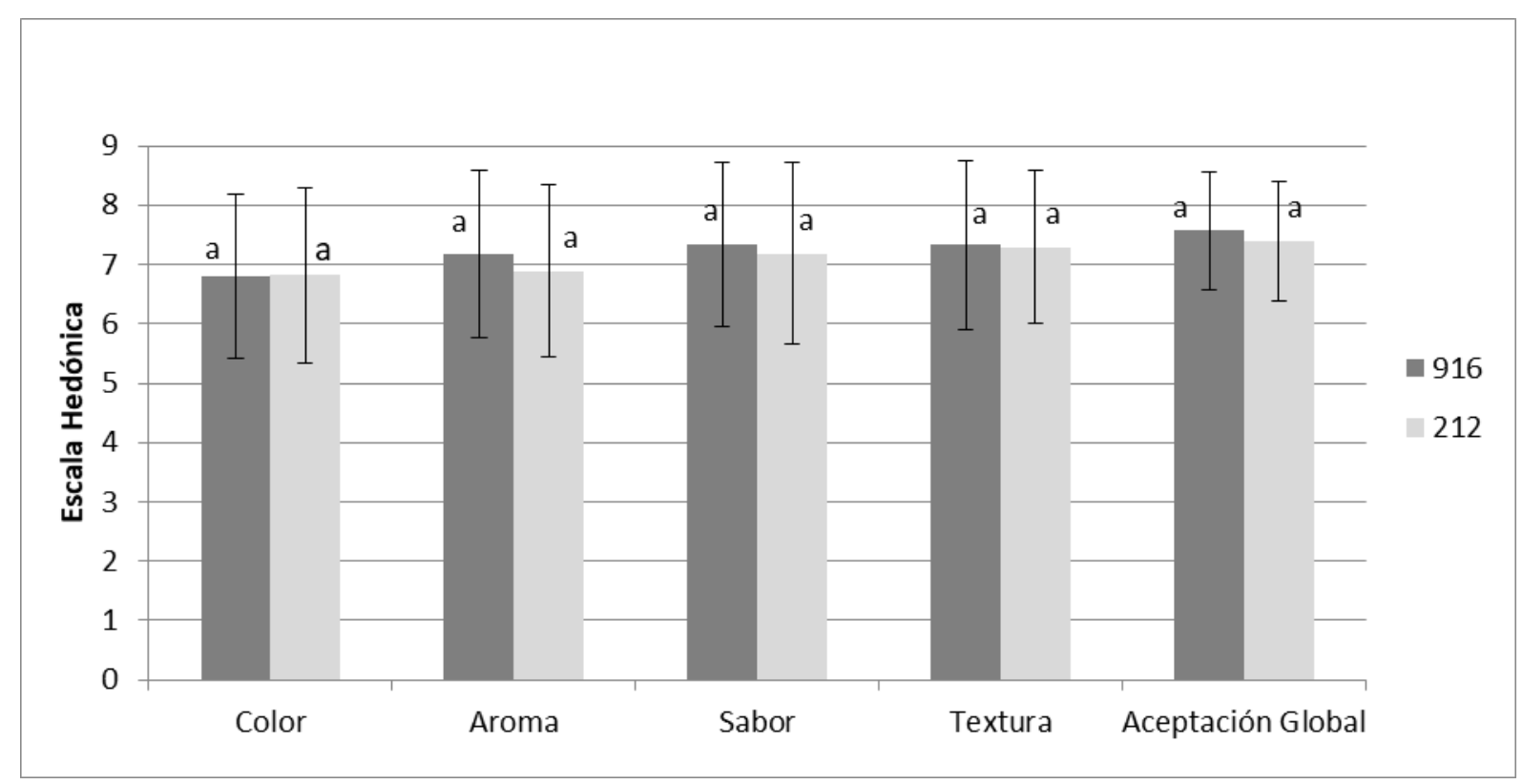

Fig. 3: Resultados de la Evaluación Sensorial (Letras distintas para cada atributo indica diferencia significativa $(p \leq 0.05)$

\section{Análisis Proximal}

Los resultados obtenidos de las dos formulaciones optimizadas se encuentran en la Tabla 6 . En la norma NTE INEN 2945 se encuentran diferentes requisitos que debe cumplir un pan. La humedad debe tener un mínimo del $20 \%$ y un máximo del $40 \%$, en las dos formulaciones (916 y 212 ), la humedad oscila entre el $33 \%$, por lo que se cumple con la norma. En relación con la grasa, los panes deben presentar un mínimo de 1.5\% y máximo de 4\% (INEN, 2014). En las dos formulaciones este valor esta alrededor del 2\%. Por último, la cantidad mínima de proteína que debe contener es de $7 \mathrm{~g} / 100 \mathrm{~g}$ y ambas formulaciones contienen cerca de $10 \mathrm{~g} / 100 \mathrm{~g}$ de producto.

Tabla 6: Análisis proximal de las formulaciones óptimas (Letras distintas en la misma línea indican diferencia significativa, $p \leq 0.05$ ).

\begin{tabular}{|l|l|l|}
\hline & 916 & 212 \\
\hline Grasa (g) & $2.21 \pm 0.14^{\mathrm{a}}$ & $2.27 \pm 0.14^{\mathrm{a}}$ \\
\hline Sodio (mg) & $499.01 \pm 1.00^{\mathrm{a}}$ & $499.03 \pm 0.96^{\mathrm{a}}$ \\
\hline Carbohidratos Totales $(\mathrm{g})$ & $45.98 \pm 0.23^{\mathrm{a}}$ & $45.71 \pm 0.25^{\mathrm{a}}$ \\
\hline Fibra (g) & $5.00 \pm 0.00^{\mathrm{a}}$ & $5.00 \pm 0.00^{\mathrm{a}}$ \\
\hline Proteína (g) & $9.98 \pm 0.02^{\mathrm{a}}$ & $10.09 \pm 0.06^{\mathrm{a}}$ \\
\hline Humedad (\%) & $33.56 \pm 0.12^{\mathrm{a}}$ & $33.55 \pm 0.12^{\mathrm{a}}$ \\
\hline Cenizas (g) & $3.27 \pm 0.08^{\mathrm{a}}$ & $3.28 \pm 0.03^{\mathrm{a}}$ \\
\hline
\end{tabular}

\section{Análisis del alveolado}

La estructura de la miga se basa mayoritariamente en el número y tamaño de los alveolos, consecuentemente los parámetros de textura dependen directamente de las características de este (número de alveolos, perímetro, circularidad, área, porcentaje de aire). La medida del perímetro se refiere a la constancia del contorno alveolar donde, mientras menor sea este valor, mayor regularidad tendrán en las áreas alveolares. Los resultados obtenidos también pueden informar sobre la capacidad de las masa para retener el aire y estos parámetros dependen de la matriz formada entre el gluten y el almidón (Correa, 2012). Tanto en los resultados obtenidos para el área total de la rodaja de las dos formulaciones, así como el perímetro no existen diferencias significativas (Tabla 7). Esto permite inferir que ambas formulaciones serán similares para el volumen específico, resultado también presentado por la Tabla 5, de la función de deseabilidad, la cual demostró que para ambas optimizaciones no existe diferencia entre el volumen específico de las dos formulaciones de panes 212 y 916 .

Por otro lado, en la circularidad, número de alveolos por $\mathrm{cm}^{2}$, tamaño medio de los alveolos y el área media de los alveolos de las dos formulaciones presentan diferencias significativas, siendo que el pan 212 presentó menor circularidad, mayor número de alvéolos y menor tamaño medio de los alveolos, indicando que esta muestra presentaría una textura más suave (pan menos firme) (Amorim et al, 2017). Cuanto mayor sea el valor de la circularidad, más redondeado es el alvéolo y, consecuentemente, mayor fuerza será necesaria para la deformación de la estructura cilíndrica del alvéolo. La muestra 212 presenta un alveolado más ovalado 
(Tabla 7), consecuentemente, menor fuerza será necesaria para deformar la miga del pan. Esta menor firmeza puede ser explicada también por el mayor número y el menor tamaño de los alvéolos (Silveira y Schmiele, 2018).

Como el número de alvéolos es mayor en el pan 212, la matriz polimérica del complejo coloidal presente entre los alvéolos es de menor espesor, favoreciendo la menor fuerza para deformar la miga del pan. Estos resultados permiten concluir que los parámetros obtenidos en el Mixolab no presentan influencia en el volumen específico de los panes, sin embargo, optimizando los parámetros termomecánicos de la masa, se podría obtener una miga con mayor suavidad.

Tabla 7: Parámetros del análisis de la miga de pan de zapallo y pan control (Letras distintas en la misma línea indican diferencia significativa entre las muestras $(p<0.05)$.

\begin{tabular}{|l|c|c|}
\hline Parámetros & 212 & 916 \\
\hline Área total de la rodaja (cm2) & $70.29 \pm 1.25^{\mathrm{a}}$ & $67.80 \pm 0.86^{\mathrm{a}}$ \\
\hline Perímetro (cm) & $54.79 \pm 4.51^{\mathrm{a}}$ & $52.71 \pm 0.80^{\mathrm{a}}$ \\
\hline Circularidad, & $0.79 \pm 0.42^{\mathrm{b}}$ & $0.83 \pm 0.01^{\mathrm{a}}$ \\
\hline Número de alveolos por cm ${ }^{2}$ & $139.56 \pm 6.22^{\mathrm{a}}$ & $123.47 \pm 2.87^{\mathrm{b}}$ \\
\hline Tamaño medio de los alvéolos $\left(\mathrm{mm}^{2}\right)$ & $5.50 \pm 0.18^{\mathrm{b}}$ & $6.68 \pm 0.28^{\mathrm{a}}$ \\
\hline Área media de los alveolos $(\%)$. & $35.76 \pm 0.81^{\mathrm{b}}$ & $38.41 \pm 0.83^{\mathrm{a}}$ \\
\hline
\end{tabular}

\section{CONCLUSIONES}

Un mayor tiempo de cocción y valores medios de enzima GO influyeron positivamente en el aumento de la estabilidad. C2 incrementó, con valores medios de GO y un mayor tiempo de cocción. C4 fue mayor al tener valores medios de GO y valores mínimos de tiempo de cocción. C5-C4 disminuyó cuando aumentaba el tiempo de cocción y valores medios de GO. El volumen específico aumentó a valores más bajos de GO y un mayor tiempo de cocción. El contenido de almidón resistente aumentó cuando incrementa el tiempo de cocción y a valores medios de GO. En relación con el color instrumental el valor de $L^{*}$ disminuye a medida que se tiene menor tiempo de cocción y menor cantidad de enzima. Con la ayuda de la función de deseabilidad fue posible obtener dos tratamientos óptimos tomando en cuenta las variables de estabilidad, C2, C4, C5-C4, Volumen Específico, Almidón Resistente y $L^{*}$. Estas formulaciones fueron obtenidas con 19.19 min de cocción y 8.9 ppm de GO y con 20 min de cocción y 11.4 ppm de GO, con una deseabilidad de 0.82 y 0.71 , respectivamente. No se obtuvo diferencia significativa en ninguno de los atributos evaluados sensorialmente (color, aroma, sabor, textura y apariencia) y las dos formulaciones fueron aceptadas con un valor de 7/9 en la escala hedónica que significa "Me gusta". En el análisis proximal tampoco existió diferencia significativa entre las dos formulaciones. Se logró obtener un pan de molde con un mejor contenido nutricional, debido al aumento del almidón resistente mediante la cocción de zapallo y se determinó que el efecto de la enzima GO depende de la cantidad utilizada y el tipo de harina.

\section{REFERENCIAS}

Amaorim, M. y otros tres autores, Amidos Modificados por Tratamento Térmico não Convencional Melhoram Qualidade da Massa e de Pão Sem Glúten, Memorias del II Simposio de Engenharia de Alimentos da Universidade Federal de Minas Gerais (II SIMEALI/UFMG): Sustentabilidade: Uma nova perspectiva na produção de alimentos, Montes Claros, Minas Gerais, Brasil (2017)

Alava, C., Desarrollo del Proceso y Caracterización de la Harina de Zapallo y Formulación de Subproductos, Tesis de Titulación, Departamento de Ingeniería en Alimentos, Escuela Superior Politécnica del Litoral, Guayaquil, Ecuador (2007)

Badui, S., Química de los Alimentos, 5 a Ed., 20-21, Pearson Educación, Ciudad de México, Mexico (2013)

Birt, D. F., Resistant Starch: Promise for Improving Human Health, doi: 10.3945/an.113.004325, Adv. Nutr., 4(6), 587-601 (2013)

Bock, J.E.,Improving and Tailoring Enzymes for Food Quality and Functionality, Enzymes in Breadmaking, Woodhead Publishing Series in Food Science, Technology and Nutrition, 181-198, Ontario, Canada (2015)

Castelli, E., Ensayos comparativos de sustitutos del bromato de potasio para su uso en panificación, Invenio, ISSN: 03293475, 5(8), 133-140 (2002)

Correa, M.J., Efecto de Celulosas Modificadas y Pectinas Sobre la Microestructura y Atributos de Calidad de la Masa Panaria, (Tesis de Doctorado), Universidad Nacional de La Plata, La Plata, Argentina (2012)

Derringer, G. y R. Suich, Simultaneous Optimization of Several Response Variables, doi: 10.1080/00224065.1980.11980968, J. Qual. Technol., 12(4), 214-219 (1980)

FAO, Crops, (en la web: http://www.fao.org/faostat/en/\#data/QC, acceso: 15 de enero de 2017)(2016)

Flecha, M., Procesos y Técnicas de Panificación (en la web: https://bit.ly/2KGkTwb, acceso: 07 de diciembre de 2017) (2015) 
Freire, W. y otros nueve autores, Encuesta Nacional de Salud y Nutrición del Ecuador, Resumen Ejecutivo. Tomo 1, 4585, Quito,Ecuador (2013)

Fu, Z., J. Chen y otros tres autores, Effect of Food Additives on Starch Retrogradation: A Review, doi:10.1002/star.201300278, Starch, 67(1-2),69-78 (2015)

Hasjim, J., Y. Ai, y J.L. Jane, Novel applications of amylose-lipid complex as resistant starch type 5; Resistant starch sources, applications and health benefits, IFT Press, (pp. 79-94), Chichester, United Kingdom (2013)

INEC, Anuario de estadísticas vitales, nacimientos y defunciones 2014 (2014)

Kundu, H., R. Bala Grewal y otros tres autores, Effect of Incorporation of Pumpkin (Curcubita moshcata) Powder and Guar Gum on the Rheological Properties of Wheat Flour, doi:10.1007/s13197-012-0777-x, J. Food Sci. Technol, 51(10), 26002607 (2012)

Loor, K., Efecto de Varios Ciclos de Enfriamiento/Calentamiento en el Contenido de Almidón Resistente Tipo III en Almidones de Achira (Canna edulis Ker) y Papa (Solanum tuberosum), Revista Bolivariana de Química, ISSN: 0250-5460, 30(1), 84-90 (2008)

Meléndez, A., I. Vicario y F. Heredia, Estabilidad de los Pigmentos Carotenoides en los Alimentos; Arch. Latinoam. Nutr., 57(2), 209-215 (2004)

Myers. R., D. Montgomery y C. Anderson-Cook, Response Surface Methodology, $4^{\text {ta }}$ ed., Wiley, New Jersey, USA (2016) NTE INEN 2945. Pan.Requisitos, 1-4, Quito-Ecuador (2014)

Onyango, C., Starch and Modified Starch in Bread Making: A Review, doi: 10.5897/AJFS2016.1481, Afr. J. Food Sci., 10(12), 344-351 (2016)

Pineda, S., Utilización de la Harina de Malanga (Xanthosoma sagittifolium) en la Obtención de Productos de Panificación; Tesis de Titulacion, Facultad de Ciencias de la Ingeniería, Universidad Tecnologica Equinoccial, Quito-Ecuador (2013)

Rey, E., Producción y Consumo de Zapallo de las Familias del Canton Sigchos en el Marco de la Seguridad Alimentaria, Tesis de Titulación, Departamento de Nutricion Humana y Dietética, Pontificia Universidad Católica del Ecuador, QuitoEcuador (2016)

Rodriges, M. y A. lemma, Planejamento de Experimentos e Otimização de Procesos. $1^{\text {a }}$ Ed., Casa do Pao Editora, Campinas, Brasil (2005)

Rodríguez-Ambriz, S., J. Islas-Hernández y otros tres autores, Characterization of a Fibre-Rich Powder Prepared by Liquefaction of Unripe Banana Flour, doi: 10.1016/j.foodchem.2007.10.007, Food Chem., 107(4),1515-1521 (2007)

Rodríguez-Manrique, J., A. Alvis-Bermudez y C. Cohen-Manrique, Análisis de Perfil de Textura de Ahuyama (Cucurbita maxima) sometida a Freído Atmosférico por Inmersión, doi: 10.4067/S0718-07642018000400055, Inf. Tecnol., 29(4), 55 64 (2018)

Rosell, C., The Science of Doughs and Bread Quality; Flour and Breads and their Fortification in Health and Disease Prevention, $1^{\text {a }}$ Ed., Elsevier, 5-12, San Diego, USA, (2011)

Silveira, M.P. y M. Schmiele, Influência nas características tecnológicas e atividade antifúngica de condimentos em pó adicionados em pães de forma. Memorias del IV Congresso Mineiro de Engenharia de Alimentos, Lavras, Minas Gerais, Brasil, (2018)

Steffolani, M., Efecto de las enzimas pentonasas, glucosa oxidasa y transglutaminasa en productos de panificación, Tesis Doctoral, Departamento de Ciencias Biológicas, Universidad Nacional de la Plata, Buenos Aires-Argentina (2010)

Vemulapalli, V., K. Miller y R. Hoseney, Glucose Oxidase in Breadmaking Systems, doi:10.1094/CCHEM.1998.75.4.439, Cereal Chem., 75(4), 439-442. (1998)

Wang, M., Effect of Pentosans on Gluten Formation and Properties, Tesis de Titulación, Wageningen University, Güeldres Países Bajos (2003)

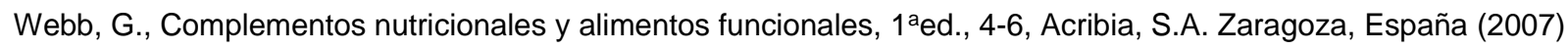

